\title{
The U-type Platform Multi-objective Topology Optimization Research
}

\author{
Chuan Jiang ${ }^{1,2,3, a, b}$, Hongyi Hü ${ }^{1,2}$, Zhizhou Xü ${ }^{1,2}$, Ruzhen Liu ${ }^{1,2}$ \\ ${ }^{1}$ Institute of optics and electronics, Chinese Academy of sciences, Chengdu610209,China \\ ${ }^{2}$ Key Laboratory of Optical Engineering, Chinese Academy of Sciences, Chengdu610209,China \\ ${ }^{3}$ University of Chinese Academy of Sciences, Beijing100039,China
}

a751906994@qq.com, ${ }^{b} 13281889539 @ 163 . c o m$

\section{Keywords:U-type Platform; multi-objective; topology optimization}

\begin{abstract}
In order to provide an effective design method which can comprehensive consider working conditions for the U-type Platform,multi-objective topology optimization was studied,based on the theory of variable density.In this optimization, volume fraction as constraints, natural frequency and stiffness were treated as optimization objectivesincluding multiple targets. In general, it is moreeasy for us to realize single objective optimization, so multi-objective optimization function which actually is a single objective function was defined. At last ,based on this function, the U-type realized optimization: Quality reduced, stiffness increased, natural frequency meet the requirements.
\end{abstract}

\section{Introduction}

In this paper, the U-type platform was used for electric-optic load which should have good dynamic and static characteristics of the structure[1,2]. So it needs to be optimized. Due to the complexity of structure condition, a single objective optimization method is difficult to comprehensively consider each working condition, then themulti-objective topology optimization method with more comprehensive consideration of various conditions was put forward.

General structure optimization hassize optimization, shape optimization and topology optimization.Compared with the former two methods of optimization, topology optimization is more broad prospects.Size optimization and shape optimization, which is under the condition of the structural layout is set to adjust the size and shape of the structure, the change is limited, the optimization effect is limited.And topology optimization can early in the design find the best force transmission path ordistribution of material in a given space to provide a conceptual design[3]. Topology optimization effect is more apparent, also has theoretical significance and application value.At present, the research of structure topology optimization are mainly concentrated on the topology optimization problem of the single target, there is few of multi-objective topology optimization.But in practical engineering there is a lot of multi-objective topology optimization problem[4]. Therefore only a single objective topology optimization is hard to achieve the optimal topology structure which meeting the needs of actual engineering. Although some scholars have studied the multi-objective problem, most focus on the frame structure topology optimization problems. For example, Wen-jieFan who focused on the frame studied the multi-objective optimization, static stiffness and dynamic vibration frequency taken as objective[5]; Lin-hua Liu use Optistruct software to optimize a suv frame and achieved the structure meeting the requirement of stiffness and vibration frequency[6]; Jin-xing Jiang also successfully got the optimized structure of dump truck framethrough multi-objective optimization. The examples above all are the successful applications of multi-objective topology optimization, and applied in practice. So it is feasible to apply multi-objective topology optimization.Apart from the above examples, this paper focused on the U-type platformwhich is more difficult to achieve a feasible optimized structure.

In this paper, based on the variable density method, multi-objective function was defined. With the use of multi-objective function, volume fraction as constraints, natural frequency and stiffness treated as optimization objectives, the performance of U-type Platform was increased.Quality reduced, stiffness increased, natural frequency meet the requirements. 


\section{Topology optimization theory}

Topology optimization that is commonly used in topology expression form and material interpolation model methods are:homogenization method, the density method, variable thickness method, the topological function description method.And the variable density method is more popular mechanics modeling approach.At present, many large CAE softwarehave optimization modulebased on variable density method, such as Hyperworks, Ansys,Nastran. In this paper, Hyperworks was used. Variable density method takes the cell density of each unit in finite element model space as design variables.The density changes between 0 and 1.After optimization, unit density is 1 (or close to 1) means the materials of the unit location at that point is very important, need to keep;Cell density is zero (or close to zero) meansthematerial of that location is not important, can be removed. Through the variable density method,the structurecan realize the efficient utilization of materials. In order to better illustrate the variable density method, an example was given.

$\min _{1 \leq j \leq p}\left(C_{j}\left(\rho_{1}, \rho_{2}, \cdots, \rho_{i}\right)\right)$, s.t. $\frac{\sum_{i=1}^{n} \rho_{\mathrm{i}} \rho_{0} V_{0 i}}{\mathrm{~m}_{0}} \leq \alpha, 0 \leq \rho_{i} \leq 1$

Where $C_{j}$ stands for compliance; $\rho_{i}$ is the cell density; $\alpha$ stands for quality percentage

\section{Multi-objective topology optimization}

In this paper,frequency and stiffness were regarded as optimization objectives, including two or more targets. So it is need to find a multi-objective function to combine frequency topologyoptimization function and stiffness topology function.

\subsection{Natural frequency topology optimization function}

Natural frequency topology optimization can maximize the frequency which can help structure avoid or higher than vibration frequency and prevent the occurrence of resonance phenomena. In the process of structural frequency optimization, if only the first order frequency as the optimization goal, when it becomes max, several other frequencies will be reduced to a lower value, the frequency aliasing phenomenon occurs. So in this paper,the average frequency optimization function was applied [8], as follows:

$\max f(\rho)=f_{0}+\alpha\left(\sum_{i=1}^{m} \frac{w_{i}}{f_{i}(\rho)-f_{0}}\right)^{(-1)}$

$f_{0}$ a given initial frequency; $m$ stands for the largest order of frequency; $w_{i}$ is the weight coefficient.

\subsection{Stiffness topology optimization function}

Stiffness topology optimization is to maximize the stiffness of the structure under constraints. Due to complex working conditions of the U-type Platform, a single objective optimization of stiffness cannot meet our requirements.In order to make the stiffness under various working conditions can be optimized, it is need to define a multi-stiffness topology optimization function. In this paper, Optistruct module of Hyperworks we used which has no stiffness response, so we used compliance response instead of stiffness. Then the function shows as follows:

$$
\min C(\rho)=\left\{\sum_{k=1}^{m} w_{k}^{q}\left[\frac{C_{k(\rho)}-C_{k}^{\min } q^{\frac{1}{q}}}{C_{k}^{\max }-C_{k}^{\min }}\right]\right\}
$$

wher $C$ stands for the compliance; $m$ is the serial number of the working condition?, $q$ is Penalty factor, $q \geq 2$.

\subsection{Multi-objective topology optimization function}

To the U-type platform with electro-optic load, the stability of the structure is very important. So it is meaningful to improve the stiffness and natural frequency.In order to realize the optimization of stiffness and natural frequency at the same time, the idea of The compromise programming method was applied which can harmoniously combine natural frequency topology optimization function and stiffness topology optimization function. And the volume fraction as constraints .Then the 
multi-objective function shows:

$$
\min F(\rho)=\left\{w^{2}\left[\sum_{k=1}^{m} w_{k} \frac{c_{k}(\rho)-c_{k}^{\min }}{c_{k}^{\max }-c_{k}^{\min }}\right]+(1-w)^{2}\left(\frac{f_{\max }-f(\rho)}{f_{\max }-f_{\min }}\right)^{2^{\frac{1}{2}}}, \frac{v(\rho)}{v_{0}} \leq \varepsilon\right.
$$

where $F$ is the muti-objective function value.

\section{4 .Multi-objective topology optimization of U-type platform}

From some air equipment, the U-type platform is the important support structure.The performance of the structure has great effect on the normal implementation of the equipment. So it is necessary to optimize the Platform.Before the topology optimization design of the platform, we should build the finite element model of the U-type platform, define related material properties, determine load and boundary condition, and establish the corresponding working condition of load analysis. The finite element model (Select 4 nodes tetrahedron units, divided into 223833)built in Optistruct shows in figure 1.The material selected High strength aluminum alloy; and working conditions uses 5 kinds of equivalent static conditions and modal condition.

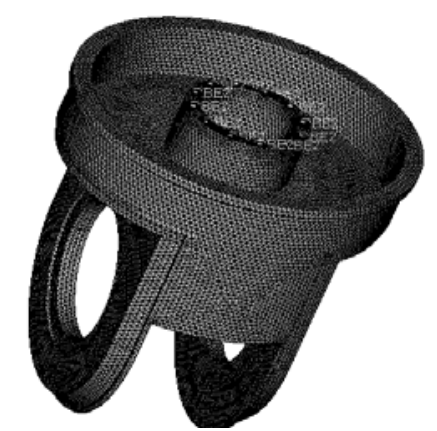

Fig.1. FEM of platform

After the load was appliedand the constraint condition of topology optimization was carried out on the platform [9], the topology optimization begins.

Step 1: define the design variable area.Choose U platform base as a design area, each unit of the relative density as design variables.In order to make the design of topology structure is easy to manufacture, design of base area to add draft manufacturing constraints, determine the direction of the draft and Angle;And set the minimum size constraints, avoiding the emergence of board structure; Set the symmetry constraint, conform to the processing and manufacturing.

Step 2: Define the structural response.Set all the complianceresponseof equivalent static conditions, frequency response, and volume fraction response.And according to the formula (3) define the user custom response which will be optimization objective.

Step 3:Define the objective function.Custom response from the formula (3) as the optimization goal, calculate the target of minimum.

Step 4:Define constraints.Select volume fraction as constraint conditions, make the optimized volume fraction shall not be greater than a value (0.55in this paper).

After defining optimization problem, the finite element model was solved usingOptistruct software.Under the premise that the calculation results of convergence, after 64 iterations, the analysis of the topology optimization results are obtained, as shown in figure 2. When the density threshold is 0.3 , the structure shows as figure 3.Multi-objective topology optimization function iteration curve as shown in figure 3 and the objective function values tend to be stable at 45 steps. 


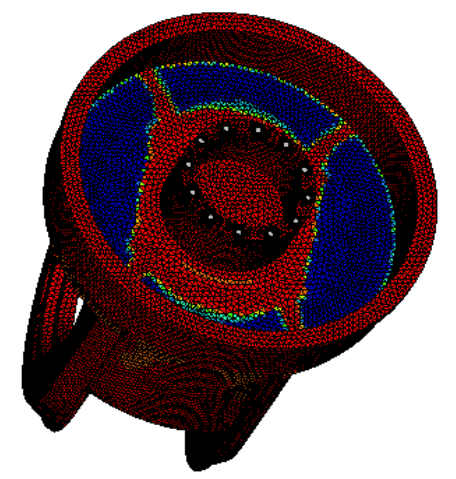

Fig.2.Model resultsof topology optimization

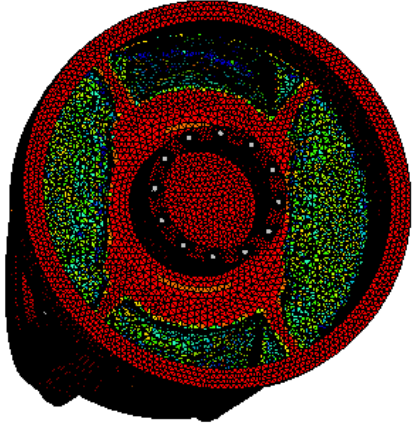

Fig.3. Model results when the density is 0.3

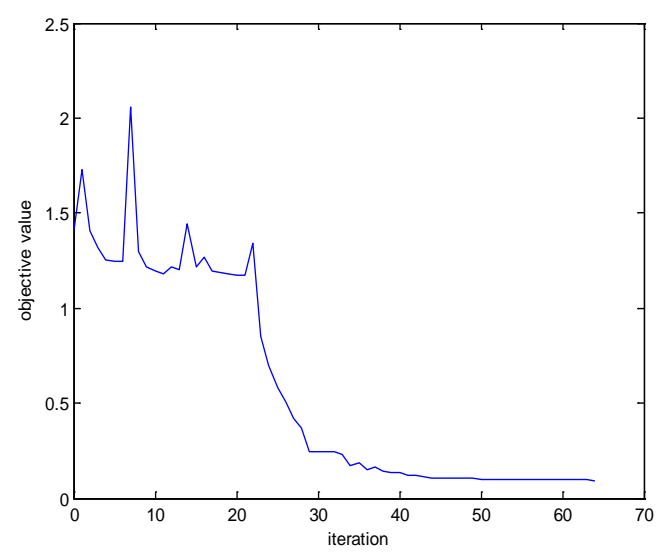

Fig.4.Objective function optimization curve

From figure 3, we can see clear power transmission path which can help us to build a new model.Then the new model shows in figure 5.

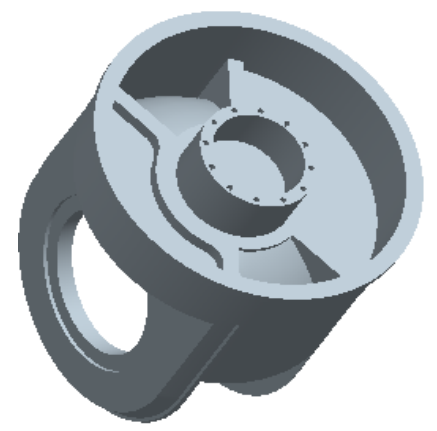

Fig.5. Optimized model of the platform

\section{Performance contrast of original and optimized model}

In order to verify the validity of the optimization design, the performance of original and optimized model should be compared. Through static analysis and modal analysis, the results show as bellows. 
Tab 1 Displacement contrast of the original model and optimized model

\begin{tabular}{|c|c|c|c|}
\hline \multirow[b]{2}{*}{$\begin{array}{c}\text { serial } \\
\text { number }\end{array}$} & \multicolumn{2}{|c|}{ Max displacement/mm } & \multirow[b]{2}{*}{$\begin{array}{c}\text { Displacement } \\
\text { decrease (\%) }\end{array}$} \\
\hline & $\begin{array}{c}\text { Original } \\
\text { model }\end{array}$ & $\begin{array}{l}\text { Optimized } \\
\text { model }\end{array}$ & \\
\hline 1 & 0.1267 & 0.01345 & 89.4 \\
\hline 2 & 0.1203 & 0.01225 & 89.82 \\
\hline 3 & 0.09821 & 0.01696 & 82.73 \\
\hline 4 & 0.08215 & 0.01411 & 82.82 \\
\hline 5 & 0.1287 & 0.01449 & 88.74 \\
\hline
\end{tabular}

Note: The serial number corresponds to the working condition

Tab2 Contrast of Frequency

\begin{tabular}{ccc}
\hline \multirow{2}{*}{$\begin{array}{c}\text { Frequency } \\
\text { order }\end{array}$} & \multicolumn{2}{c}{ Frequency $/ \mathrm{Hz}$} \\
\cline { 2 - 3 } & $\begin{array}{c}\text { Original } \\
\text { model }\end{array}$ & $\begin{array}{c}\text { Optimized } \\
\text { model }\end{array}$ \\
\hline 1 & 310.79 & 348.85 \\
\hline 2 & 439.57 & 438.03 \\
\hline 3 & 454.37 & 443.73 \\
\hline 4 & 858.77 & 797.89 \\
\hline
\end{tabular}

Tab.3 Contrast of mass

\begin{tabular}{llc}
\hline \multicolumn{2}{c}{ Mass $/ \mathrm{kg}$} & $\begin{array}{c}\text { Mass decrease } \\
(\%)\end{array}$ \\
$\begin{array}{llc}\text { Original } \\
\text { model }\end{array}$ & $\begin{array}{l}\text { Optimized } \\
\text { model }\end{array}$ & 15.986 \\
\hline 6 & 4.94 & 1586 \\
\hline
\end{tabular}

From the table 1, under various equivalent staticconditions, stiffness of the model is enhanced. For example,in serial number 1,the model of maximum displacement decreased from $0.1267 \mathrm{~mm}$ to $0.01345 \mathrm{~mm}$, stiffness got obvious improvement.From the table 2, the first order natural frequency increased from $310.79 \mathrm{~Hz}$ to $348.85 \mathrm{~Hz}$ which can help the U-type structure Have a good dynamic stiffness. From table 3, the mass decrease from $6 \mathrm{~kg}$ to $4.94 \mathrm{~kg}$ which realizes the lightweight design.

\section{Summary}

Through combining stiffness topology optimization and frequency topology optimization, the multi-objective topology optimization function was achieved. With the multi-objective topology optimization function used, the U-type platform reached the optimization goal. Quality reduced, stiffness increased, natural frequency meet the requirements.So it is feasible to use multi-objective topology optimization to improve the performance of the structure which can comprehensive consider working conditions.

\section{References}

[1] Zhuangxinyu, Chen Zhaobing, Movement frame structure design and analysis on some designing pod, Infrared and Laser Engineering.43(2014) 3444-3450.

[2] WANG Ping, ZHANG Guoyu ,LIU Jia, Topology Optimization Design for Inner Frame of AirborneElectro-optical Platform, JOURNAL OF MECHANICAL ENGINEERING. 50(2014)135-141.

[3] Xia Tianxing, Yao Weixing, A Survey of Topology Optimization of Continuum Structure, ADVANCES IN AERONAUTICAL SCIENCE AND ENGINEERING. 2(2012)1-11.

[4]ZHAN JinQing, ZHANG XianMin, RESEARCH ON STATIC AND DYNAMIC 
MULTI-OBJECTIVE TOPOLOGYOPTIMIZATION OF CONTINUUM STRUCTURES, Journal of Mechanical Strength.32(2010) 933-937.

[5] Fan Wenjie, Fan Zijie, Su Ruiyi, Research on Multi -objective Topology Optimization on Bus Chassis Frame, CHINA MECHANICAL ENGINEERING.19(2007)1505-1507.

[6]Liu Linhua, Xin Yong, Wang Wei, Multi-objective Topology Optimization for an Off-road VehicleFrame Based on Compromise Programming ,Mechanical Science and Technology for Aerospace Engineering.30(2011) 382-385.

[7]Jiang Jinxing, GuZhengqi, Multi-objective Topology Optimization of a Mining Dump Truck Frame,CHINA MECHANICAL ENGINEERING.24(2013)1028-1032.

[8]Cheng, Z.-D.M.N.K.H.-C, Topological design for vibrating structures,Comput.Methods Appl.Mech.Engrg.121(1995) 259-280.

[9]LV Jianli, YAO Shouwen, Study on the Method of Bearing Loads Defining in Strength Analysis of Transmission Case, Vehicle \& Power Technology.(2011) 39-42. 